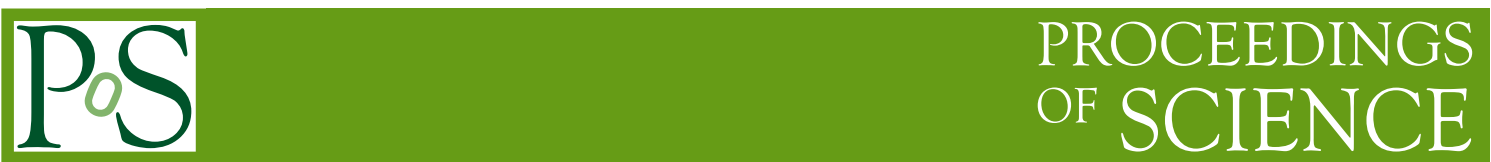

\title{
Electroweak physics at a future EIC
}

\author{
Krishna Kumar*t \\ Department of Physics, University of Massachusetts, Amherst, MA 01002, USA \\ E-mail: kkumar@physics.umass.edu
}

\begin{abstract}
We discuss potential electroweak measurements that represent a new opportunity from the potentially large luminosities that are envisioned in design studies of a polarized lepton light ion collider (EIC). At integrated luminosities of greater than $10 \mathrm{fb}^{-1}$ and center of mass energies between 10 and $100 \mathrm{GeV}$, it becomes possible to measure semi-leptonic weak neutral current amplitudes mediated by the $\mathrm{Z}^{0}$ boson via electroweak interference measurements in a collider environment for the first time. We explore the new physics topics that are opened up due to precision measurements of these weak neutral current amplitudes, with the inclusion of longitudinal polarization in both the lepton and hadron beams. One can also achieve new sensitivity in the search for lepton flavor violation in $e-\tau$ conversion, and explore novel aspects of quark densities in nuclei in e-A collisions.
\end{abstract}

XVIII International Workshop on Deep-Inelastic Scattering and Related Subjects April 19 -23, 2010

Convitto della Calza, Firenze, Italy

\footnotetext{
* Speaker.

$\dagger$ funded by US Department of Energy, Division of Nuclear Physics, and Brookhaven National Laboratory
} 


\section{Introduction}

Nearly 50 years ago, soon after the discovery of parity violation in beta decay, Zel'dovich speculated that there might be an analogous parity violating neutral current interaction [1]. He noted that if such an interaction existed, then parity violation would be manifested in lepton-nucleon scattering due to the interference between the weak and electromagnetic amplitudes. He predicted that if one scatters longitudinally polarized electrons off unpolarized protons and flipped the sign of the beam polarization, the fractional difference in the cross-section would be:

$$
A_{\mathrm{PV}} \equiv \frac{\sigma_{R}-\sigma_{L}}{\sigma_{R}+\sigma_{L}} \simeq \frac{\left|A_{Z}\right|}{\left|A_{\gamma}\right|} \simeq \frac{G_{F} Q^{2}}{4 \pi \alpha} \simeq 10^{-4} Q^{2}
$$

For typical fixed target experiments, $A_{P V}$ ranges from roughly $10^{-4}$ to as small as $10^{-7}$. In the mid-seventies, parity violation in deep inelastic electron nucleon scattering was first observed at SLAC [2], from which the electron-quark weak neutral current coupling could be extracted. The measurement was an important validation of the Standard Model, and the extracted value of the electroweak mixing angle $\sin ^{2} \theta_{W}$ matched the corresponding value obtained from neutral current neutrino scattering experiments.

Over the past 20 years, the experimental techniques employed to measure these tiny left-right asymmetries have been steadily refined such that statistical errors and systematic errors approaching a few parts per billion (ppb) are possible [3]. Depending on the choice of target and kinematic variables, this has facilitated measurements in several important physics topics, such as many-body nuclear physics, nucleon structure and searches for physis beyond the standard model at the $\mathrm{TeV}$ scale.

\section{Parity-Violating Deep Inelastic Scattering}

$A_{P V}$ in DIS can be written as

$$
\begin{gathered}
A_{P V}=Q^{2} \frac{G_{F}}{2 \sqrt{2} \pi \alpha}\left[a(x)+\frac{1-(1-y)^{2}}{1+(1-y)^{2}} b(x)\right], \\
a(x) \equiv \Sigma_{i} f_{i}(x) C_{1 i} q_{i} / \Sigma_{i} f_{i}(x) q_{i}^{2} \\
b(x) \equiv \Sigma_{i} f_{i}(x) C_{2 i} q_{i} / \Sigma_{i} f_{i}(x) q_{i}^{2} .
\end{gathered}
$$

Here, $C_{1 i}\left(C_{2 i}\right)$ are the weak vector(axial-vector) weak charges for the $i$ th quark flavor, $x$ is the fraction of the nucleon momentum carried by the struck quark, $f_{i}(x)$ are parton distribution functions and $q_{i}$ are the electromagnetic charges. The $a(x)$ term arises from the product of the electron axial-vector coupling and the quark vector coupling and is typically the dominant term. For an isoscalar target such as deuterium, the dependence on structure largely cancels out in the $A_{P V}$ ratio of the weak and electromagnetic amplitudes:

$$
\begin{gathered}
a(x)=\frac{6}{5}\left[\left(C_{1 u}-\frac{1}{2} C_{1 d}\right)+\text { corrections }\right] \\
b(x)=\frac{6}{5}\left[\left(C_{2 u}-\frac{1}{2} C_{2 d}\right) \frac{q(x)-\bar{q}(x)}{q(x)+\bar{q}(x)}+\text { corrections }\right],
\end{gathered}
$$


where $q(x)=u(x)+d(x)$. For scattering off the proton,

$$
a(x)=\left[\frac{u(x)+0.91 d(x)}{u(x)+0.25 d(x)}\right] .
$$

In trying to make precision measurements of $A_{\mathrm{PV}}$ in PVDIS, several important issues should be taken under consideration. The $a(x)$ term is a factor of 5 to 10 larger than the $b(x)$ term because the latter involves the vector coupling of the electron, which is small in the electroweak theory. In trying to test the electroweak theory, it is important to be able to extract the value of the electroweak mixing angle $\sin ^{2} \theta_{W}$ with a relative accuracy better than $1 \%$ in order to be relevant in constraining new physics at the $\mathrm{TeV}$ scale. Consequently, $A_{\mathrm{PV}}$ must be measured to $1 \%$ relative accuracy or better. In order to avoid uncertainties from sea-quark distributions and higher-twist effects, it is important to have $Q^{2} \geq 2 \mathrm{GeV}^{2}$ and $x \geq 0.35$. The DIS cross-section is steeply falling as $x$ increases. The above factors have precluded a followup precision measurement to E122 for the past two decades.

At a polarized electron light ion collider (EIC), where longitudinally polarized 4 to $20 \mathrm{GeV}$ electrons would collide with 60 to $250 \mathrm{GeV}$ polarized light ions, many of the hadronic physics issues are alleviated. In addition, because data can be obtained over a wide range of $Q^{2}$ and $x$ with high precision, it would be easy to separately measure $a(x)$ and $b(x)$, with very little impact of higher-twist effects and axial-current uncertainties. Indeed, it might be possible to directly measure higher-twist effects at fixed $x$ given the luminosity and kinematic reach of the EIC. In particular, it has emerged [4] that the higher-twist coefficient for the parity-violating asymmetry off ${ }^{2} \mathrm{H}$ is dynamically interesting and isolates a quark-quark correlator that might ultimately be calculable on the lattice.

\section{Target Spin-Flip PV Asymmetries}

While the EIC would make modest improvements on fixed target electroweak measurements planned for Jefferson Laboratory with a $12 \mathrm{GeV}$ beam, it is with target-flip parity-violating asymmetries that the EIC offers an entirely new opportunity. In order to describe the physics, it is instructive to review the most general hadronic tensor that is compatible with Lorentz and CP invariance. It is well-known that, if $\mathrm{P}$ conservation is additionally imposed, there are four structure functions, $F_{1}, F_{2}$ in the unpolarized case, and $g_{1}, g_{2}$ with the inclusion of lepton and quark spin degrees of freedom. If P-violation is allowed, then there are additionally four new structure functions: $F_{3}, g_{3}, g_{4}$ and $g_{5}$. This phenomenology has been explored by many authors $[5,6,7,8]$.

The EIC will have sufficient luminosity so that the weak-electromagnetic interference amplitude can be extracted from the parity-violating asymmetry in the collisions of unpolarized electrons off longitudinally polarized light ions, At sufficiently high $Q^{2}$ and $W^{2}$, one can extract two independent new structure functions $g_{1}^{\gamma Z}$ and $g_{5}^{\gamma Z}$, which will provide unique new information about polarized parton densities in the nucleon, augmenting existing measurements in electromagnetic double-spin asymmetries.

Studies of $Q^{2}$ evolution of these new structure functions could potentially provide new insights into the QCD structure of the nucleon. Cumulatively, it is envisioned that the EIC will collect data with three polarized light ion species: ${ }^{1} \mathrm{H},{ }^{2} \mathrm{H}$ and ${ }^{3} \mathrm{He}$. It will be possible to measure both single- 
and double-spin asymmetries with longitudinal polarization in the neutral current process, as well as analogous asymmetries in the charged current process, thus accessing electroweak amplitudes with $\gamma$ exchange, $W$ exchange and $\gamma-Z$ interference. Putting all these structure functions together would provide stringent new tests of the QCD structure of the nucleon and help alleviate the limiting systematic errors from poor knowledge of the electron and light-ion longitudinal polarizations.

\section{Lepton-Flavor Violation}

With the observation of neutrino oscillations and thus the indirect but incontrovertible evidence for neutrino mass, it has become increasingly important to search for anomalous amounts of charged lepton flavor violation. Virtually any new model that tends to predict new dynamics at the $\mathrm{TeV}$ scale at the Large Hadron Collider (LHC) also tends to predict charged lepton flavor violation that is significantly enhanced over the miniscule standard model rate. In the muon sector, new and ambitious high flux experiments are being launched to look for the rare processes $\mu \rightarrow e \gamma$ and $\mu+N \rightarrow e+N$. For the third generation $\tau$ lepton, the best limits tend to come from B-factory data.

With a large integrated luminosity, one can search for the analogous process $e+N \rightarrow \tau+X$ at an EIC. It has been recently pointed out [9] that this process might provide complementary information to searches for lepton flavor violation in $\tau$ decays, thus further constraining phenomenological models of new physics at the $\mathrm{TeV}$ scale. Such searches might take on added significance if particular topologies of new physics signals are obtained at the LHC or if charged lepton flavor violation is observed in the muon sector.

Experimentally, the envisioned detector capabilities around the lepton-hadron interaction point at the EIC should make it particularly suitable to search for the rare $e-\tau$ conversion process. Apart from precision tracking and calorimetry, the detector is expected to have a vertex detector encircling the collision. Using topological cuts and displaced vertices, it should be feasible to find events where the electron converted to a $\tau$ lepton at the DIS scattering process without producing any neutrinos. Studies are ongoing to determine the ultimate sensitivity. Coupled with further theoretical work, it should be possible to determine how unique and important would be the discovery reach of this process.

\section{Parity Violation in DIS off Heavy Nuclei}

One other new opportunity at the EIC will be to study parity violation in DIS in e-A collisions. In analogy to the unpolarized light ion case, nuclear structure functions $F_{2}$ and $F_{3}$ can be measured. Because of the different strengths of the weak neutral current coupling between u- and d-quarks, these new structure functions carry a different flavor-dependence compared to the corresponding electromagnetic structure functions.

Even after more than two decades of theoretical and experimental effort, there is no consensus on the dynamical origins of the EMC effect. Flavor separation of nuclear structure functions would provide important new insight into this fundamental topic. Recently, quarks in nuclei have emerged as an important aspect of interpreting the result of an electroweak measurement i.e. the comparison of the standard model prediction for the ratio of charged to neutral current amplitude in neutrino 
deep inelastic scattering in the $\mathrm{NuTeV}$ experiment. It has been pointed out that the $\mathrm{NuTeV}$ result would be consistent with the standard model if one invokes an isovector-vector mean field that shifts the quark distributions. The size of the effect grows with neutron or proton excess. However, this experimental signature is indistinguishable from charge symmetry violation in the nucleon.

At an EIC, one would have the opportunity to study this by comparing the electroweak asymmetry in parity-violating deep inelastic scattering of polarized electrons off unpolarized light ions to that scattering of unpolarized heavy ions. A difference between the two measured asymmetries would be unambiguous evidence for a new aspect of the EMC effect and could provide important new information to constrain theoretical models. Such measurements are likely more interpretable than double-spin asymmetry measurements with polarized heavy ions, since such targets are hard to produce and it is difficult to evaluate the degree of polarization.

\section{Conclusion}

The advent of a polarized electron light ion collider could potentially open up a new era of precision electroweak measurements. Apart from searches for physics beyond the standard model in the weak neutral current sector, the new data could probe novel aspects of charged lepton flavor violation, higher twist effects, polarized structure functions and the EMC effect.

\section{Acknowledgement}

It is a pleasure to thank I. Cloet, A. Deshpande, W. Marciano, K. Paschke, M. Ramsey-Musolf, P. Souder and Werner Vogelsang for useful discussion and feedback.

\section{References}

[1] Ya. B. Zel'dovich,J.Exptl.Theoret.Phys. (U.S.S.R.), 36, 1959, pp. 964-966.

[2] C. Y. Prescott et.al., Phys.Lett., B84, 524, (1979).

[3] K. S. Kumar and P. A. Souder, Prog.Part.Nucl.Phys., 45, 2000, pp. S333-S395.

[4] S. Mantry, M. J. Ramsey-Musolf and G. F. Sacco, arXiv:1004.3307 [hep-ph], and references therein.

[5] M. Anselmino, A. Efremov and E. Leader, Phys. Rept. 261, 1 (1995) [Erratum-ibid. 281, 399 (1997)] [arXiv:hep-ph/9501369].

[6] X. D. Ji, Nucl. Phys. B 402, 217 (1993).

[7] M. Stratmann, A. Weber and W. Vogelsang, Phys. Rev. D 53, 138 (1996) [arXiv:hep-ph/9509236].

[8] T. Hobbs and W. Melnitchouk, Phys. Rev. D 77, 114023 (2008) [arXiv:0801.4791 [hep-ph]].

[9] M. Gonderinger and M. J. Ramsey-Musolf, arXiv:1006.5063 [hep-ph].

[10] I. C. Cloet, W. Bentz and A. W. Thomas, Phys. Rev. Lett. 102, 252301 (2009) [arXiv:0901.3559 [nucl-th]]. 